\title{
NEUMANN BOUNDARY VALUE PROBLEM FOR SYSTEM OF EQUATIONS OF NON-EQUILIBRIUM SORPTION
}

\author{
I.A. KALIEV, G.S. SABITOVA
}

\begin{abstract}
Filtration of liquids and gases containing associated (dissolved, suspended) solids in porous media is accompanied by diffusion of these substances and mass transfer between the liquid (gas) and solid phases. In this work, we study the system of equations modeling the process of a non-equilibrium sorption. We prove an existence and uniqueness theorem for a multi-dimensional Neumann initial-boundary value problem in the Hölder classes of functions. We obtain a maximum principle, which plays an important role in the proof of the theorem. The uniqueness of the solution follows this principle. The existence of a solution to the problem is shown by Schauder fixed point theorem for a completely continuous operator; we describe a corresponding operator. We obtain estimates ensuring the complete continuity of the constructed operator and the mapping of some closed set of functions into itself over a small time interval. Then we obtain the estimates allowing us to continue the solution up to arbitrary finite time.
\end{abstract}

Keywords: modeling of process of non-equilibrium sorption, Neumann initial boundary value problem, global unique solvability.

Mathematics Subject Classification: 35Q35, 76S05

\section{INTRODUCTION}

Almost all liquids in the nature are solutions, that is, they are mixture of two or more substances. The filtration of liquids and gases in porous media containing associated (dissolved, suspended) solids is accompanied by diffusion of these substances and mass transfer between the liquid (gas) and solid phases. The most common types of mass transfer are sorption and desorption, ion exchange, dissolution and crystallization, mudding, sulfation and suffusion, waxing. Taking into consideration the features of physical and chemical interaction of solutions with reservoir rocks, the problems of equilibrium and non-equilibrium are considered.

In the work we prove a global unique solvability of the Neumann initial-boundary value problem modelling the process of a non-equilibrium sorption.

\section{Formulation of PROBlem}

Let $m(x, t)$ be the porosity of a medium, $0<m(x, t) \leqslant 1$; the porous space be filled by a solution and by a precipitated solid phase, $c(x, t)$ be the mass concentration of a certain substance in the liquid phase (per unit of the volume), $s(x, t)$ be the mass concentration of the precipitated solid of this substance (per unit of the volume of the pores).

Under equilibrium conditions when the contact between the solution and the solid is kept for a long time, the relation between the concentrations $c(x, t)$ in the solution and on the sorbate $s(x, t)$ is determined by the sorption isotherm. For small concentrations of the solution, the absorption value is determined by a linear dependence, namely, by Henry isotherm $s=\Gamma c$, where $\Gamma>0$ is some constant depending on physical and chemical properties of the medium (Henry constant).

I.A. Kaliev, G.S. Sabitova, Neumann boundary value problem for system of equations of NON-EQUILIBRIUM SORPTION.

(C) Kaliev I.A., Sabitova G.S. 2019.

Submitted August 7, 2018. 
Equilibrium sorption equation can not always completely characterize the features of sorption and substances exchange in a two-phase system solution-solid. In works [1]-3] there were proposed mathematical models describing non-equilibrium sorption processes. At that, the concentration $s$ of solid stage is related with the concentration $c$ in the liquid stage by the equation

$$
\frac{\partial s}{\partial t}=\frac{1}{\tau}(\Gamma c-s),
$$

where a positive constant $\tau$ is a character relaxation time, $G$ is the Henry constant. The concentration $c$ of a substance in a solution satisfies the equation

$$
m \frac{\partial c}{\partial t}=D \triangle c-v \nabla c-\frac{\partial s}{\partial t}
$$

where $D(x, t)>0$ is the diffusion coefficient, $v(x, t)$ is the filtration rate vector, which are regarded as known functions of the mentioned arguments; $\Delta$ is the Laplace operator, $\nabla$ is the gradient, $v \cdot \nabla c$ is the scalar produce of the vectors $v$ and $\nabla c$.

In work [4], a global unique solvability was proved for the Dirichlet initial-boundary problem for system (1)-(2). In [5], 6] there was formulated a difference approximation of the differential problem by an implicit scheme, a solution to the difference problem was constructed by means of the sweep method and the results of the numerical experiments were presented.

In this work we consider the Neumann boundary value problem for system of equations (1)-(2) describing the process of a non-equilibrium sorption.

Let $\Omega$ be a bounded domain in a $n$-dimensional space $\mathbb{R}^{n}$ with a sufficiently smooth boundary $S=\partial \Omega, Q_{T}=\Omega \times(0, T), T>0, S_{T}=S \times(0, T)$ be the lateral surface of cylinder $Q_{T}$. We need to find functions $c(x, t), s(x, t)$ defined in domain $Q_{T}$ satisfying equations (1), (2) in $Q_{T}$, obeying the initial conditions

$$
\begin{aligned}
& c(x, 0)=c_{0}(x), \\
& s(x, 0)=s_{0}(x),
\end{aligned}
$$

and the Neumann boundary condition

$$
\frac{\partial c(x, t)}{\partial n}=0,(x, t) \in S_{T}
$$

where $n$ is the outward normal to $S=\partial \Omega$.

The main result of the work is the following theorem.

Theorem 1. Let the coefficients $m, D, v$ of equation (2) belong the Hölder space $C^{\alpha, \alpha / 2}\left(\bar{Q}_{T}\right)$, $0<\alpha<1$; the boundary of the domain $S$ be $C^{2+\alpha}$-smooth; the functions $c_{0}(x), s_{0}(x)$ belong to the spaces $C^{2+\alpha}(\bar{\Omega}), C^{\alpha}(\bar{\Omega})$, respectively ; the matching conditions

$$
\frac{\partial c_{0}(x)}{\partial n}=0, \quad x \in S
$$

hold as well as the conditions $0 \leqslant c_{0}(x) \leqslant M, 0 \leqslant s_{0}(x) \leqslant \Gamma M, x \in \Omega$. Then problem (1)-(5) possesses a unique classical solution $c(x, t) \in C^{2+\alpha, 1+\alpha / 2}\left(\bar{Q}_{T}\right), s(x, t) \in C^{\alpha, 1+\alpha / 2}\left(\bar{Q}_{T}\right)$ and the estimates hold:

$$
0 \leqslant c(x, t) \leqslant M, \quad 0 \leqslant s(x, t) \leqslant \Gamma M, \quad(x, t) \in Q_{T} .
$$

\section{Proof of Theorem}

We begin with proving estimates providing the maximum principle:

$$
\begin{array}{lll}
0 \leqslant c(x, t) \leqslant M, & (x, t) \in Q_{T}, \\
0 \leqslant s(x, t) \leqslant \Gamma M, & (x, t) \in Q_{T} .
\end{array}
$$

Equation (1) and condition (3) imply the representation

$$
s(x, t)=s_{0}(x) e^{-t / \tau}+\frac{\Gamma}{\tau} e^{-t / \tau} \int_{0}^{t} c(x, \theta) e^{\theta / \tau} d \theta .
$$


Substituting (8) into (2), we obtain:

$$
m \frac{\partial c}{\partial t}-D \Delta c+v \cdot \nabla c+\frac{\Gamma}{\tau} c=\frac{1}{\tau} s_{0}(x) e^{-t / \tau}+\frac{\Gamma}{\tau^{2}} e^{-t / \tau} \int_{0}^{t} c(x, \theta) e^{\theta / \tau} d \theta .
$$

Assume that a negative minimum $c_{\min }<0$ of the function $c(x, t)$ is attained at some point $\left(x_{0}, t_{0}\right)$ inside the domain $Q_{T}$. Then at this point we have

$$
c_{t} \leqslant 0, \quad-\Delta c \leqslant 0, \quad \nabla c=0,
$$

and by (9) we get:

$$
\begin{aligned}
& \frac{\Gamma}{\tau} c_{\min } \geqslant \frac{1}{\tau} s_{0}\left(x_{0}\right) e^{-t_{0} / \tau}+\frac{\Gamma}{\tau^{2}} c_{\min } e^{-t_{0} / \tau} \int_{0}^{t_{0}} e^{\theta / \tau} d \theta, \\
& \Gamma c_{\min } \geqslant s_{0}\left(x_{0}\right) e^{-t_{0} / \tau}+\Gamma c_{\min } e^{-t_{0} / \tau}\left(e^{t_{0} / \tau}-1\right), \\
& 0 \geqslant s_{0}\left(x_{0}\right) e^{-t_{0} / \tau}-\Gamma c_{\min } e^{-t_{0} / \tau},
\end{aligned}
$$

and hence, we arrive at a contradiction since $s_{0}(x) \geqslant 0$, while $c_{\min }<0$. Therefore, a negative minimum of the function $c(x, t)$ can not be attained inside the domain $Q_{T}$.

On the boundary $S_{T}$ the minimum can not be attained by condition (5) and the following ZarembaGiraud lemma.

Lemma 1. Let

$$
L u=\sum_{i, j=1}^{n} a_{i, j}(x) u_{x_{i} x_{j}}(x)+\sum_{i=1}^{n} b_{i}(x) u_{x_{i}}(x)
$$

be an elliptic operator in an unbounded domain $\Omega$ with a sufficiently smooth boundary, $u \in C^{2}(\Omega) \cap$ $C^{1}(\bar{\Omega}), L u \leqslant 0$ in $\Omega$ and let the function $u(x)$ attain its proper global maximum in a boundary point $x_{0} \in \partial \Omega$. Then

$$
\left.\frac{\partial u}{\partial n}\right|_{x_{0}}<0
$$

where $n$ is the outward normal to $S=\partial \Omega$ at the point $x_{0}$.

For harmonic functions, this lemma was proved by Zaremba [7], and in a more general formulation it was proved by Giraud [8].

In our case

$$
L c=D \Delta c-v \cdot \nabla c=F(x, t)=m \frac{\partial c}{\partial t}+\frac{\Gamma}{\tau} c-\frac{1}{\tau} s_{0}(x) e^{-t / \tau}-\frac{\Gamma}{\tau^{2}} e^{-t / \tau} \int_{0}^{t} c(x, \theta) e^{\theta / \tau} d \theta .
$$

Assume that the negative minimum $c_{\min }<0$ of the function $c(x, t)$ is attained at some point $\left(x_{0}, t_{0}\right)$ in the boundary of the domain $S_{T}$. Then

$$
\begin{aligned}
F\left(x_{0}, t_{0}\right) & =m\left(x_{0}, t_{0}\right) \frac{\partial c}{\partial t}\left(x_{0}, t_{0}\right)+\frac{\Gamma}{\tau} c_{\min }-\frac{1}{\tau} s_{0}\left(x_{0}\right) e^{-t_{0} / \tau}-\frac{\Gamma}{\tau^{2}} e^{-t_{0} / \tau} \int_{0}^{t_{0}} c\left(x_{0}, \theta\right) e^{\theta / \tau} d \theta \\
& \leqslant m\left(x_{0}, t_{0}\right) \frac{\partial c}{\partial t}\left(x_{0}, t_{0}\right)+\frac{\Gamma}{\tau} c_{\min }-\frac{1}{\tau} s_{0}\left(x_{0}\right) e^{-t_{0} / \tau}-\frac{\Gamma}{\tau^{2}} c_{\min } e^{-t_{0} / \tau} \int_{0}^{t_{0}} e^{\theta / \tau} d \theta \\
& =m\left(x_{0}, t_{0}\right) \frac{\partial c}{\partial t}\left(x_{0}, t_{0}\right)+\frac{\Gamma}{\tau} c_{\min }-\frac{1}{\tau} s_{0}\left(x_{0}\right) e^{-t_{0} / \tau}-\frac{\Gamma}{\tau} c_{\min } e^{-t_{0} / \tau}\left(e^{t_{0} / \tau}-1\right) \\
& =m\left(x_{0}, t_{0}\right) \frac{\partial c}{\partial t}\left(x_{0}, t_{0}\right)-\frac{1}{\tau} s_{0}\left(x_{0}\right) e^{-t_{0} / \tau}+\frac{\Gamma}{\tau} c_{\min } e^{-t_{0} / \tau}<0 .
\end{aligned}
$$


Therefore, $F\left(x, t_{0}\right)<0$ in the vicinity of the point $x_{0}$ and we can apply Zaremba-Giraud lemma, that is,

$$
\left.\frac{\partial c}{\partial n}\right|_{x_{0}}<0 .
$$

This contradicts boundary condition (5).

Hence, the function $c(x, t)$ attains its minimum on the lower boundary of the domain $Q_{T}$, that is, at initial time and at this time, the function $c_{0}(x)$ is non-negative. Thus, we have proved that $c(x, t) \geqslant 0$, $(x, t) \in Q_{T}$.

Assume now that the function $c(x, t)$ attains its positive maximum $c_{\max }>M$ inside the domain $Q_{T}$, that is, there exists a point $\left(x_{1}, t_{1}\right) \in Q_{T}$ such that $c\left(x_{1}, t_{1}\right)=c_{\max }>M$. At this point we have

$$
c_{t} \geqslant 0, \quad-\Delta c \geqslant 0, \quad \nabla c=0,
$$

and by (9) we get the inequalities

$$
\begin{aligned}
& \frac{\Gamma}{\tau} c_{\max } \leqslant \frac{1}{\tau} s_{0}\left(x_{1}\right) e^{-t_{1} / \tau}+\frac{\Gamma}{\tau^{2}} c_{\max } e^{-t_{1} / \tau} \int_{0}^{t_{1}} e^{\theta / \tau} d \theta, \\
& \Gamma c_{\max } \leqslant s_{0}\left(x_{1}\right) e^{-t_{1} / \tau}+\Gamma c_{\max } e^{-t_{1} / \tau}\left(e^{t_{1} / \tau}-1\right), \\
& 0 \leqslant s_{0}\left(x_{1}\right) e^{-t_{1} / \tau}-\Gamma c_{\max } e^{-t_{1} / \tau}=\left(s_{0}\left(x_{1}\right)-\Gamma c_{\max }\right) e^{-t_{1} / \tau} .
\end{aligned}
$$

We again have arrived at a contradiction, since $s_{0}(x) \leqslant \Gamma M$ and $c_{\max }>M$.

At the boundary $S_{T}$, the maximum can not be attained by condition (5) and Zaremba-Giraud lemma. We consider

$$
L c=D \Delta c-v \cdot \nabla c=F(x, t)=m \frac{\partial c}{\partial t}+\frac{\Gamma}{\tau} c-\frac{1}{\tau} s_{0}(x) e^{-t / \tau}-\frac{\Gamma}{\tau^{2}} e^{-t / \tau} \int_{0}^{t} c(x, \theta) e^{\theta / \tau} d \theta
$$

Assume that a positive maximum $c_{\max }>M$ of the function $c(x, t)$ is attained at some point $\left(x_{1}, t_{1}\right)$ in the boundary of the domain $S_{T}$. Then

$$
\begin{aligned}
F\left(x_{1}, t_{1}\right) & =m\left(x_{1}, t_{1}\right) \frac{\partial c}{\partial t}\left(x_{1}, t_{1}\right)+\frac{\Gamma}{\tau} c_{\max }-\frac{1}{\tau} s_{0}\left(x_{1}\right) e^{-t_{1} / \tau}-\frac{\Gamma}{\tau^{2}} e^{-t_{1} / \tau} \int_{0}^{t_{1}} c\left(x_{1}, \theta\right) e^{\theta / \tau} d \theta \\
& \geqslant \frac{\Gamma}{\tau} c_{\max }-\frac{1}{\tau} s_{0}\left(x_{1}\right) e^{-t_{1} / \tau}-\frac{\Gamma}{\tau^{2}} c_{\max } e^{-t_{1} / \tau} \int_{0}^{t_{1}} e^{\theta / \tau} d \theta \\
& =\frac{\Gamma}{\tau} c_{\max }-\frac{1}{\tau} s_{0}\left(x_{1}\right) e^{-t_{1} / \tau}-\frac{\Gamma}{\tau} c_{\max } e^{-t_{1} / \tau}\left(e^{t_{1} / \tau}-1\right) \\
& =-\frac{1}{\tau} s_{0}\left(x_{1}\right) e^{-t_{1} / \tau}+\frac{\Gamma}{\tau} c_{\max } e^{-t_{1} / \tau} \\
& =\frac{1}{\tau}\left(\Gamma c_{\max }-s_{0}\left(x_{1}\right)\right) e^{-t_{1} / \tau}>\frac{1}{\tau}(\Gamma M-\Gamma M) e^{-t_{1} / \tau}=0 .
\end{aligned}
$$

Therefore, $F\left(x, t_{1}\right)>0$ in the vicinity of the point $x_{1}$ and we can apply Zaremba-Giraud lemma for the case of the maximum and hence

$$
\left.\frac{\partial c}{\partial n}\right|_{x_{1}}>0 .
$$

This contradicts boundary condition (5).

Thus, the function $c(x, t)$ attains its maximum on the lower boundary of the domain $Q_{T}$, that is, at the initial time; at this time we have the estimate $c_{0}(x) \leqslant M$. Therefore, $c(x, t) \leqslant M,(x, t) \in Q_{T}$. This completes the proof of estimate (6).

Estimate (7) is implied by representation (8) with employing (6). Indeed, since $s_{0}(x) \geqslant 0, c(x, t) \geqslant 0$, it follows from (8) that $s(x, t) \geqslant 0$ for all $(x, t) \in Q_{T}$. 
Since $s_{0}(x) \leqslant \Gamma M, c(x, t) \leqslant M$, then

$$
s(x, t) \leqslant s_{0}(x) e^{-t / \tau}+\frac{\Gamma M}{\tau} e^{-t / \tau} \int_{0}^{t} e^{\theta / \tau} d \theta \leqslant \Gamma M e^{-t / \tau}+\Gamma M e^{-t / \tau}\left(e^{t / \tau}-1\right)=\Gamma M .
$$

This completes the proof of estimate (7).

The uniqueness of solution to problem (1)-(5) follows Lemma.

We proceed to the proof of the existence of a solution to problem (1)-(5); we shall do this by means of the Schauder theorem on a fixed point of a completely continuous operator. We denote by $V_{T_{1}}$ the following closed convex subset in $C^{2+\alpha, 1+\alpha / 2}\left(\bar{Q}_{T_{1}}\right)$ :

$$
V_{T_{1}}=\left\{\tilde{c}(x, t) \mid \tilde{c}(x, 0)=c_{0}(x) ; \frac{\partial \tilde{c}(x, t)}{\partial n}=0,(x, t) \in S_{T_{1}} ;\|\tilde{c}\|_{C^{2+\alpha, 1+\alpha / 2}\left(\bar{Q}_{T_{1}}\right)} \leqslant K\right\},
$$

where $K$ is some fixed positive number depending on data of problem (1)-(5), which we define later. Given a function $\tilde{c} \in V_{T_{1}}$, we find a function

$$
\tilde{s}(x, t)=s_{0}(x) e^{-t / \tau}+\frac{\Gamma}{\tau} e^{-t / \tau} \int_{0}^{t} \tilde{c}(x, \theta) e^{\theta / \tau} d \theta .
$$

Now to each function $\tilde{c} \in V_{T_{1}}$, we associate a function $c=\Lambda(\tilde{c})$ as a solution to the problem

$$
\begin{aligned}
& m \frac{\partial c}{\partial t}-D \Delta c+v \cdot \nabla c+\frac{\Gamma}{\tau} c=\frac{1}{\tau} \tilde{s}, \\
& c(x, 0)=c_{0}(x), \quad x \in \Omega ; \quad \frac{\partial c(x, t)}{\partial n}=0, \quad(x, t) \in S_{T_{1}} .
\end{aligned}
$$

Let us prove that the operator $\Lambda$ is completely continuous and for sufficiently small $T_{1}$, it maps the set $V_{T_{1}}$ into itself.

First we are going to show that $\tilde{s} \in C^{\alpha, \alpha / 2}\left(\bar{Q}_{T_{1}}\right)$. Identity (10) implies the inequality

$$
|\tilde{s}|_{Q_{T_{1}}}^{(0)} \equiv \max _{(x, t) \in \bar{Q}_{T_{1}}}|\tilde{s}(x, t)| \leqslant\left|s_{0}\right|_{\Omega}^{(0)}+\Gamma|\tilde{c}|_{Q_{T_{1}}}^{(0)} \max _{t \in\left[0, T_{1}\right]}\left(1-e^{-t / \tau}\right) .
$$

Expanding the function $e^{-t / \tau}$ into the Maclaurin series, it is easy to obtain the following estimate as $T_{1}<\tau:$

$$
|\tilde{s}|_{Q_{T_{1}}}^{(0)} \leqslant\left|s_{0}\right|_{\Omega}^{(0)}+T_{1} \frac{\Gamma}{\tau}|\tilde{c}|_{Q_{T_{1}}}^{(0)} .
$$

In the same, identity (10) implies the estimate

$$
\begin{aligned}
|\tilde{s}|_{x, Q_{T_{1}}}^{(\alpha)} & \equiv \sup _{(x, t),\left(x^{\prime}, t\right) \in \bar{Q}_{T_{1}}} \frac{\left|\tilde{s}(x, t)-\tilde{s}\left(x^{\prime}, t\right)\right|}{\left|x-x^{\prime}\right|^{\alpha}} \\
& \leqslant\left|s_{0}\right|_{x, \Omega}^{(\alpha)}+\Gamma|\tilde{c}|_{x, Q_{T_{1}}}^{(\alpha)} \max _{t \in\left[0, T_{1}\right]}\left(1-e^{-t / \tau}\right) \leqslant\left|s_{0}\right|_{x, \Omega}^{(\alpha)}+T_{1} \frac{\Gamma}{\tau}|\tilde{c}|_{x, Q_{T_{1}}}^{(\alpha)},
\end{aligned}
$$

that is, the function $\tilde{s}$ satisfies the Hölder condition in the spatial variable with an exponent $\alpha$.

The function $\tilde{s}$ satisfies the Hölder condition in the variable $t$ with arbitrary exponent $0<\beta \leqslant 1$ being even a Lipschitz function since it possesses a bounded in time derivative:

$$
\begin{aligned}
& \tilde{s}_{t}(x, t)=-\frac{1}{\tau} s_{0}(x) e^{-t / \tau}-\frac{\Gamma}{\tau^{2}} e^{-t / \tau} \int_{0}^{t} \tilde{c}(x, \theta) e^{\theta / \tau} d \theta+\frac{\Gamma}{\tau} \tilde{c}(x, t), \\
& \left|\tilde{s}_{t}\right|_{Q_{T_{1}}}^{(0)} \leqslant \frac{1}{\tau}\left|s_{0}\right|_{\Omega}^{(0)}+\frac{\Gamma}{\tau}|\tilde{c}|_{Q_{T_{1}}}^{(0)} \max _{t \in\left[0, T_{1}\right]}\left(1-e^{-t / \tau}\right)+\frac{\Gamma}{\tau}|\tilde{c}|_{Q_{T_{1}}}^{(0)} \leqslant \frac{1}{\tau}\left|s_{0}\right|_{\Omega}^{(0)}+\frac{2 \Gamma}{\tau}|\tilde{c}|_{Q_{T_{1}}}^{(0)} .
\end{aligned}
$$

In particular, as $\beta=1$, we get

$$
\frac{\left|\tilde{s}(x, t)-\tilde{s}\left(x, t^{\prime}\right)\right|}{\left|t-t^{\prime}\right|^{\alpha / 2}\left|t-t^{\prime}\right|^{1-\alpha / 2}} \leqslant\left|\tilde{s}_{t}\right|_{Q_{T_{1}}}^{(0)} .
$$


This implies the inequality

$$
|\tilde{s}|_{t, Q_{T_{1}}}^{(\alpha / 2)} \equiv \sup _{(x, t),\left(x, t^{\prime}\right) \in \bar{Q}_{T_{1}}} \frac{\left|\tilde{s}(x, t)-\tilde{s}\left(x, t^{\prime}\right)\right|}{\left|t-t^{\prime}\right|^{\alpha / 2}} \leqslant T_{1}^{1-\alpha / 2}\left|\tilde{s}_{t}\right|_{Q_{T_{1}}}^{(0)} .
$$

Estimates (13)-(16) yield that $\tilde{s} \in C^{\alpha, \alpha / 2}\left(\bar{Q}_{T_{1}}\right)$, and for $T_{1}<1$, the estimate is true:

$$
\|\tilde{s}\|_{C^{\alpha, \alpha / 2}\left(\bar{Q}_{T_{1}}\right)} \leqslant C_{1}\left\|s_{0}\right\|_{C^{\alpha}(\bar{\Omega})}+T_{1}^{1-\alpha / 2} C_{2}\|\tilde{c}\|_{C^{\alpha, \alpha / 2}\left(\bar{Q}_{T_{1}}\right)},
$$

where $C_{1}, C_{2}$ are some positive constants independent of $s_{0}$ and $\tilde{c}$. The constants $C_{1}, C_{2}$ depend on $T$, but are independent of $T_{1}<\min \{T, 1, \tau\}$.

Since

$$
\tilde{s}_{t}=\frac{1}{\tau}(\Gamma \tilde{c}-\tilde{s})
$$

we have $\tilde{s} \in C^{\alpha, 1+\alpha / 2}\left(\bar{Q}_{T_{1}}\right)$.

A solution $c(x, t)$ to problem (11), (12) obeys the estimate [9]:

$$
\|c\|_{C^{2+\alpha, 1+\alpha / 2}\left(\bar{Q}_{T_{1}}\right)} \leqslant C\left(\left\|c_{0}\right\|_{C^{2+\alpha}(\bar{\Omega})}+\|\tilde{s}\|_{C^{\alpha, \alpha / 2}\left(\bar{Q}_{T_{1}}\right)}\right),
$$

where $C$ is some positive constant independent of $c_{0}, \tilde{s}$. The constant $C$ depends on $T$ but is independent of $T_{1}<T$. Employing (17), (18), we find that

$$
\|c\|_{C^{2+\alpha, 1+\alpha / 2}\left(\bar{Q}_{T_{1}}\right)} \leqslant C_{3}\left(\left\|c_{0}\right\|_{C^{2+\alpha}(\bar{\Omega})}+\left\|s_{0}\right\|_{C^{\alpha}(\bar{\Omega})}\right)+T_{1}^{1-\alpha / 2} C_{4}\|\tilde{c}\|_{C^{\alpha, \alpha / 2}\left(\bar{Q}_{T_{1}}\right)} .
$$

This implies that the operator $\Lambda: \tilde{c} \rightarrow c$ is completely continuous.

We fix the constant $K$ involved in the definition of the set $V_{T_{1}}$ by the requirement

$$
K>C_{3}\left(\left\|c_{0}\right\|_{C^{2+\alpha}(\bar{\Omega})}+\left\|s_{0}\right\|_{C^{\alpha}(\bar{\Omega})}\right) .
$$

For the sake of definitenss we let

$$
K=2 C_{3}\left(\left\|c_{0}\right\|_{C^{2+\alpha}(\bar{\Omega})}+\left\|s_{0}\right\|_{C^{\alpha}(\bar{\Omega})}\right) .
$$

The it follows from (19) that for sufficiently small $T_{1}$, the operator $\Lambda$ maps the set $V_{T_{1}}$ into itself.

By the Schauder theorem on a fixed point of a completely continuous operator, the set $V_{T_{1}}$ contains a fixed point $\tilde{c}$, which together with the corresponding function $\tilde{s}$ in (10) solves problem (1)-(5) in the time interval $\left[0, T_{1}\right]$. In $k$ steps, the obtained solution can be continued on $\left[T_{k}, T_{k+1}\right], k=1,2, \ldots$, and $T_{k+1}-T_{k} \geqslant \delta>0$ with $\delta$ independent of the index $k$. This is seen in view of estimate (19)

$$
C_{3}\left(\left\|c_{0}\right\|_{C^{2+\alpha}(\bar{\Omega})}+\left\|s_{0}\right\|_{C^{\alpha}(\bar{\Omega})}\right)+T_{1}^{1-\alpha / 2} C_{4}\|\tilde{c}\|_{C^{\alpha, \alpha / 2}\left(\bar{Q}_{T_{1}}\right)}<K=2 C_{3}\left(\left\|c_{0}\right\|_{C^{2+\alpha}(\bar{\Omega})}+\left\|s_{0}\right\|_{C^{\alpha}(\bar{\Omega})}\right) .
$$

Since $\|\tilde{c}\|_{C^{\alpha, \alpha / 2}\left(\bar{Q}_{T_{1}}\right)} \leqslant K$, this implies that as $\delta$ we can choose

$$
\delta^{1-\alpha / 2}=\frac{K}{2 C_{4} K}=\frac{1}{2 C_{4}},
$$

which is independent of $k$. Hence, in finitely many steps, the solution can be continued to each $0<T<+\infty$.

\section{BIBLIOGRAPHY}

1. L. Lapidus, W.R. Amundson. Mathematics of adsorption in beds. VI. The effect of longitudinal diffusion in ion exchange and chromatographic columns // J. Phys. Chem. 56:8, 984-988 (1952).

2. K.H. Coats, B.D. Smith. Dead and pore volume and dispersion in porous media // Soc. Petrol. Eng. J. 4:1, 73-84 (1964).

3. P.Ya. Polubarinova-Kochina (ed.) Development of studies on filtration theory in USSR. Nauka, Moscow (1969). (in Russian).

4. I.A. Kaliev, G.S. Sabitova. On a problem of nonequilibrium sorption // Sibir. Zhurn. Industr. Matem. 6:1, 35-39 (2003). (in Russian).

5. I.A. Kaliev, S.T. Mukhambetzhanov, G.S. Sabitova. Numerical modeling of the non-equilibrium sorption process // Ufimskij Matem. Zhurn. 8:2, 39-43 (2016). [Ufa Math. J. 8:2, 39-43 (2016).] 
6. I.A. Kaliev, S.T. Mukhambetzhanov, G.S. Sabitova. Mathematical modeling of non-equilibrium sorption // Far East J. Math. Sci. 99:12, 1803-1810 (2016).

7. S. Zaremba. Sur un problème toujours possible comprenant, à titre de cas particulier, le problème de Dirichlet et celui de Neumann // J. Math. Pures Appl. 6, 127-163 (1927).

8. G. Giraud. Généralisation des problèmes sur les opérations du type elliptique // Bull. Sc. Math. II. Ser. 56. 248-272 (1932).

9. O.A. Ladyzhenskaya, V.A. Solonnikov, N.N. Ural'tseva. Linear and quasi-linear equations of parabolic type. Nauka, Moscow (1967). [Transl. Math. Monog. 23. Amer. Math. Soc., Providence, RI (1968).]

Ibragim Adietovich Kaliev,

Sterlitamak Branch of Bashkir State University,

Lenin av. 49,

453103, Sterlitamak, Russia

E-mail: kalievia@mail.ru

Gul'nara Sagyndykovna Sabitova,

Sterlitamak Branch of Bashkir State University,

Lenin av. 49,

453103, Sterlitamak, Russia

E-mail: sabitovags@mail.ru 